\title{
Phonetic categorisation and cue weighting in adolescents with Specific Language Impairment (SLI)
}

\author{
Outi Tuomainen $^{1}$, Nichola J. Stuart ${ }^{2}$, \& Heather van der Lely ${ }^{3 \dagger}$ \\ ${ }^{1}$ Department of Speech, Hearing and Phonetic Sciences, University College London, UK, ${ }^{2}$ \\ Department of Psychology, Social Work and Public Health, Oxford Brookes University, UK, \\ ${ }^{3}$ Department of Psychology, Harvard University, Cambridge, MA, USA.
}

\begin{abstract}
This study investigates phonetic categorisation and cue weighting in adolescents and young adults with Specific Language Impairment (SLI). We manipulated two acoustic cues, vowel duration and F1 offset frequency, that signal word-final stop consonant voicing ([t] and $[\mathrm{d}])$ in English. Ten individuals with SLI (14;0-21;4 years), ten age-matched controls (CA; 14;6-21;9 years) and ten non-matched Adult controls (23;3-36;0 years) labelled synthetic CVC nonwords in an identification task. The results showed that the adolescents and young adults with SLI were less consistent than controls in the identification of the good category representatives. The group with SLI also assigned less weight to vowel duration than the Adult controls. However, no direct relationship between phonetic categorisation, cue weighting and language skills was found. These findings indicate that some individuals with SLI have speech perception deficits but they are not necessarily associated with oral language skills.
\end{abstract}

$\dagger$ Deceased

Correspondence: Outi Tuomainen, Department of Speech, Hearing and Phonetic Sciences, University College London, Chandler House, 2 Wakefield Street, London WC1N 1PF, UK. Tel. +44 207679 4232. Email: o.tuomainen@ucl.ac.uk 


\section{Introduction}

Successful spoken language acquisition requires the ability to organise heterogeneous auditory input into discrete phonological categories. Different languages have different phonological units (phonemes) and acoustic realizations (allophones). When we acquire the phonological system of our first language (L1), we learn to attend to those acoustic differences which affect the meanings of words, and to ignore those acoustic differences which do not affect word meanings.

Categorical perception emerges whenever the allophonic within-category differences are compressed and the between-category differences are enhanced (Liberman, Harris, Hoffman \& Griffith, 1957). For example, it is easier to differentiate two speech sounds when the sounds belong to different phonetic categories than when they belong to the same category (e.g. the perception of the /r/-/1/ distinction by Japanese and English listeners). In traditional categorical perception experiments the effect of a single acoustic cue (e.g. Voice Onset Time (VOT), formant frequency transition, or vowel duration) for sound categorization is measured. However, speech is a complex signal consisting of multiple overlapping acoustic cues and, in real life, people are likely to utilize several different acoustic cues in speech perception (Holt \& Lotto, 2006). Despite this, the existence of multiple acoustic dimensions for speech and other auditory categories does not imply their perceptual equivalence. In specific contexts, some of these cues are considered more informative than other cues and they can be more heavily weighted when determining the phonological identity of a sound (Dorman, Studdert-Kennedy \& Raphael, 1977; Holt \& Lotto, 2006). This is referred to as cue weighting and several factors have been found to contribute to cue weighting. The presence of background noise, a change in the task demand or an attentional manipulation (such as the presence of a distractor task) can all change the perceptual strategy adopted as to which cues to employ in phonetic categorisation (Gordon, Eberhardt, \& Rueck1, 
1993; Holt \& Lotto, 2006). Furthermore, the use of these cues does not seem to be a fixed property of the perceptual system in Typical Development (TD) (Krause, 1982; Nittrouer, 2005; Nittrouer, Manning, \& Meyer, 1993; Nittrouer \& Studdert-Kennedy, 1987; Ohde, Haley, Vorperian, \& McMahon, 1995). For example, Nittrouer and Studdert-Kennedy (1987) investigated the sensitivity to acoustic variation in children and adults and found that children and adults generally make use of different cues to distinguish between phonetic contrasts. They manipulated two acoustic cues, the frequency of the friction noise and the second formant (F2) transition at the onset of the vowel, that distinguishes the fricatives: $[\mathrm{s}]$ and $\left[\int\right]$ in two different vowel contexts: [u] and [i] (i.e., $<$ sue $>,<$ shoe $>,<$ sea $>,<$ she $>$ ). They found that younger children (3-4 years of age) primarily used the vowel onset transition cue whereas older children ( 5 years of age) weight this cue slightly less. In contrast, adults and children from about 7 years of age rely more on the friction noise than on the vowel onset transition cue. This could indicate that children utilize a different perceptual unit to adults: whilst adults generally use sub-syllabic units, such as phonemes, as the central units of perception, children use larger chunks, such as syllables or words (Nittrouer, 1992; Nittrouer, Crowther \& Miller, 1998; Nittrouer \& Miller, 1997). Nittrouer and colleagues have argued that the change in perceptual strategy takes place during the first 5-7 years of life as a result of increasing linguistic experience in the L1. This Developmental Weighting Shift (DWS) hypothesis proposes that in perceiving speech segments, children generally assign more weight to the dynamic cues (such as vowel formant transitions) as they signal syllable structure whereas adults assign more weight to the static segment-intrinsic cues (such as fricative noise spectra).

Not all researchers agree with the DWS hypothesis. One alternative is Sussman's (2001) sensory hypothesis that proposes that children make use of those cues that are acoustically salient (e.g., steady-state formants) and that this can also explain the differences found in cue weighting in adults and children. Sussman (2001) argues that these 
differences are due to general sensory processing differences between children and adults (but cf. Mayo \& Turk, 2005).

However, there is general agreement that the role of cue weighting in L1 phonological acquisition and processing is important. Infants become perceptually tuned to the characteristics of L1 in their first year (Jusczyk \& Luce, 1994; Kuhl, Williams, Lacerda, Stevens \& Lindblom, 1992; Werker and Tees, 1984), but this process takes time to develop into adult like perception (Morrongiello, Robson, Best \& Clifton, 1984; Nittrouer, 2004; Nittrouer, Crowther \& Miller, 1998; Nittrouer, Miller, Crowther \& Manhart, 2000). Specifically, children appear to apply different weightings to acoustic dimensions in perceiving L1 speech categories compared to adults (Hazan \& Barrett, 2000; Mayo \& Turk, 2004; Nittrouer, 2004). For example, in languages, such as in English, in which vowel duration is a prominent cue in the voicing categorization of following final stop consonants (e.g. [t]-[d]), children tend to favour the vowel formant transitions over vowel duration (Nittrouer, 2004). However, in atypical speech and language development, some acoustic dimensions may be less heavily weighted as a consequence of weak auditory representations. For example, if they are more susceptible to noise or are represented with more variability by the auditory system than other acoustic dimensions (Nittrouer, Shune \& Lowenstein, 2011; Schwartz, Scheffler \& Lopez, 2013; Sussman, 1993).

Phonetic categorisation and cue weighting in Specific Language Impairment (SLI)

Specific Language Impairment (SLI) is a developmental disorder affecting language acquisition in otherwise TD children (Leonard, 2014). It is a heterogeneous disorder that can affect one or more components of language - syntax, morphology, phonology, pragmatics and vocabulary (lexicon). Typically it is morpho-syntax and phonology that are the most affected. 
For example, many children with SLI exhibit persisting deficits in marking verb tense (e.g. past tense '-ed') and agreement (e.g. third person singular '-s') more frequently than TD children, and difficulties in the comprehension of reversible passive sentences (e.g. 'The lorry was hit by the car'), forming WH-object questions (e.g. 'Who did John see?') and repeating nonwords (e.g. 'blonterstaping') (Leonard, 2014).

One influential account proposes that these linguistic deficits in SLI are a consequence of a more general deficit in processing rapidly changing auditory information (Tallal, 1976; Tallal \& Stark, 1981). This theory holds that a deficit in processing acoustic features that are brief or rapidly changing (e.g. formant transitions) can lead to unstable or imprecise phonological representations which, in turn, disrupt normal language learning and lead to deficits in language processing (Tallal, Miller \& Fitch, 1993). Indeed, these difficulties in discriminating rapid temporal events cuing phonological category membership (such as formant transition distinguishing the place of articulation in the [ba] - [da] contrast) have been frequently found in some (but not all) children with SLI (Tallal, 1976; Tallal \& Piercy, 1975; Tallal \& Stark, 1981).

Nevertheless, not all findings provide support for the rapid auditory processing deficit account of SLI. Sussman (1993) investigated the ability to discriminate and identify the English [ba] - [da] contrast in 5-6 year old children with SLI and their age and language matched controls and reported that the group with SLI showed relatively normal discrimination ability in the $\mathrm{CV}$ contrast but were less accurate than the controls in the identification task. The poor identification performance was particularly prominent in the most prototypical sounds (i.e. in the category representatives), as manifested by generally shallower identification slopes and greater variability in phoneme boundary placement. Sussman (1993) concluded that the main problem in SLI was not a failure to appropriately discriminate sounds but a failure in forming phonological representations and actually linking 
acoustic information to these representations. This suggests that, instead of poor sensitivity to specific acoustic features (such as formant transitions), children with SLI have an underlying deficit in encoding phonetic categories which then has an impact on speech and language processing.

A more recent study has provided further support for this view. Nittrouer and colleagues (2011) investigated auditory sensitivity and category formation in children with Phonological Processing Deficit (PPD) and their TD age-matched controls and adults. They used a "same-different" (AX) discrimination task and a phonetic labelling task and reported that children with PPD showed a similar sensitivity to spectral glides, and that they weighted formant transitions as strongly as the age-matched control children. However, the PPD group were less consistent than controls in discriminating non-speech stimuli at the "same" endpoint suggesting a difficulty in category formation for the first of the two stimuli in the AX trial.

Further evidence for impaired phonetic category formation in SLI was found in an electrophysiological and behavioural study by Shafer, Morr, Datta, Kurtzberg, and Schwartz (2005). They reported deficient speech discrimination abilities as indexed by an absent mismatch negativity (MMN) response accompanied with poor behavioural identification and relatively good behavioural discrimination of [bIt] vs. [bet] syllables in children with SLI. They concluded that children with SLI fail to correctly weight the relevant acoustic cues for phonetic identity (but cf. Coady, Kluender \& Evans, 2005). Furthermore, Schwartz et al. (2013) used a categorical perception paradigm in 6-9 year old children with SLI and their TD age-matched controls and they reported that the group with SLI needed more durational difference in vowels than their TD controls to identify word final consonant voicing. They also reported less accurate identification for category end-points (i.e. more response uncertainty) in SLI than in the TD controls and shifted category boundaries in SLI. 
They concluded that vowel duration is not as salient a cue as other available cues for children with SLI, as it is for TD children.

In summary, these results suggest that children with SLI do not have impaired or diminished sensitivity to acoustic properties in the speech signal but that they have difficulties forming coherent perceptual categories based on sensory information (Nittrouer, et al., 2011; Schwartz et al., 2013; Sussman, 1993).

In the current study we extend the above research by investigating phonetic categorisation and perceptual cue weighting in an older group of individuals (aged between 14 -21 years of age) with severe and persistent SLI. Specifically, we investigate how adolescents and young adults with SLI weight vocalic duration and formant transitions in their voicing decisions for word-final stop consonants (namely [t] and [d]) in English). An older group of individuals with SLI was chosen for the current study because previous research has shown that phoneme boundary sharpening (especially if synthetic speech is used) can continue well into the second decade of life and that children as old as 12 years of age may still not show adult-like phonetic categorisation performance (Coady et al., 2005; Hazan \& Barrett, 2000). To our knowledge, no study has investigated cue weighting in SLI.

Based on previous research we hypothesize that:

1) If adolescents with SLI have speech perception deficiencies as a result of impaired phonetic categorisation, we predict that the group with SLI will have extended areas of response uncertainty and shallower identification slopes than their TD age-matched controls.

2) If vowel duration is not as salient a cue for children with SLI as it is for TD children, the group with SLI should assign less weight to the vocalic duration than the TD age-matched 
control group. This leads to a prediction that the adolescents with SLI will have shallower identification slopes than their age-matched controls.

3) However, if adolescents with SLI have an auditory deficit in processing rapid acoustic information, we predict that the formant frequency change cue will be less salient to them. Therefore, we predict that adolescents with SLI will rely less on the formant transition cue than their TD age-matched controls and they will have smaller separation of the category boundaries of the two identification functions.

\section{Method}

The project received ethical approval from the University College London (UCL) Research ethics committee. Informed written consent was obtained from the parent/guardian and/or the participant and all gave their verbal consent to participate in the study.

\section{Participants}

A total of 30 participants divided into three groups took part in the study. The group with SLI ( $\mathrm{n}=10$, Mean age=17;0 years, range: 14;0-21;4 years, 6 male) were recruited from a larger group of language impaired children who had been participating in research at the Centre for Developmental Language Disorders and Cognitive Neuroscience (DLDCN) over a number of years. The individuals with SLI taking part in the present study had persistent language difficulties that were still present in adolescence and early adulthood (see Appendix A for individual language scores). All the participants had a formal diagnosis of SLI from Speech and Language Therapists and Educational Psychologists, as measured by standardised tests of language abilities, and either had attended or were still attending special schools/language units for children with SLI. None of them had an additional diagnosis of Attention Deficit and Hyperactivity Disorder (ADHD), Autistic Spectrum Disorder (ASD) or dyspraxia or any evidence of neurological deficits or deficits in social-emotional behaviour. 
Clinical profiles of language impaired individuals can change with age (Bishop, 1992) and some of the individuals taking part in this study were older than the ceiling age used in many of the standardised tests typically used in assessing language ability. However, prior to taking part in the study all of the participants with SLI were re-tested on a battery of standardised and non-standardised tests tapping language and cognitive ability. The standardised tests used were: one non-verbal test: Ravens Progressive Matrices (RPM; Raven, Raven, \& Court, 1998) and four core language tests, two receptive: Test for Reception of Grammar (TROG-2; Bishop, 2003) which measures grammatical comprehension of sentences; British Picture Vocabulary Scales (BPVS-II; Dunn, Dunn, Whetton \& Burley, 1997) which measures single word vocabulary, and two expressive: the recalling sentences sub-test of the Clinical Evaluation of Language Fundamentals (CELF-3; Semel, Wiig, \& Secord, 1995); Test of Word Finding (TWF-2; German, 2000) ${ }^{1}$. Participants had to have a zscore of at least -1.5 (corresponding to $-1.5 \mathrm{SD}$ ) on two or more of the standardised language tests and a non-verbal standard score of 85 or more on the RPM on their most recent test scores to be classified as SLI. Despite their age (14+ years), all participants with SLI still fulfilled this criteria except for two (S04 and S08). Furthermore, two non-standardized language tests (Verb Agreement and Tense (VATT), van der Lely, 2000; and the Test of Active and Passive Sentences (TAPS, van der Lely, 1996a) were administered as an additional inclusion criteria. This was done as the intensive therapy these individuals have received will have concentrated on specific structures measured in the standardized language tests (e.g., in TROG-2), and further investigation of those areas that are found to be particularly difficult in children with SLI is warranted. These tests tap i) syntax (the TAPS - a test of understanding reversible sentences and ii) morpho-syntax (the VATT—an expressive test of tense and agreement marking of regular and irregular verbs). Investigation of the

\footnotetext{
${ }^{1}$ BPVS-II is standardised on children up to the age of $15 ; 8$; TWF-2 up to $12 ; 11$; TROG-2 up to $16 ; 0$ CELF-3 up to $21 ; 0$ years. Where individuals were older than these cut-off ages, their raw scores on the tests were converted to standard scores at the ceiling level for the individual test.
} 
performance of TD children on these tests found that errors are rarely made after 6-8 years of age $^{2}$ (Ramus, Marshall, Rosen \& van der Lely, 2013; van der Lely, 1996b; van der Lely, 2000; van der Lely, Rosen \& McClelland, 1998) whilst children with SLI were found to make $20 \%$ or more errors. All the adolescents and young adults with SLI taking part in the current study performed poorly on two or more standardised or non-standardised language measures. Descriptive data for the group with SLI is displayed in Table 1 and details of the latest test scores used in the selection process for this study for each of the participants with SLI are reported in the Appendix.

Table 1. Summary of language and nonverbal intelligence scores for the SLI group $(\mathrm{N}=10)$.

\begin{tabular}{|l|l|l|l|l|}
\hline Test & \multicolumn{2}{|c|}{ Standard score } & \multicolumn{2}{c|}{ z-score } \\
\hline & Mean (SD) & \multicolumn{1}{c|}{ range } & \multicolumn{1}{c|}{ Mean (SD) } & \multicolumn{1}{c|}{ range } \\
\hline RPM & $96.5(6.9)$ & $87-106$ & $-0.2(0.5)$ & $-0.9-0.4$ \\
\hline BPVS-II & $72.0(8.9)$ & $59-90$ & $-1.9(0.6)$ & $-2.7-(-0.7)$ \\
\hline TROG-2 & $83.0(12.6)$ & $67-97$ & $-1.2(0.8)$ & $-2.2-(-0.2)$ \\
\hline CELF-3* & $3.3(0.5)$ & $3-4$ & $-2.2(0.2)$ & $-2.3-(-2.0)$ \\
\hline TWF-2 & $74.7(7.3)$ & $61-83$ & $-1.7(0.5)$ & $-2.6-(-1.1)$ \\
\hline VATT & $44.9(29.7)$ & $3-80$ & N/A & N/A \\
\hline TAPS-R & $56.4(11.5)$ & $40-73$ & N/A & N/A \\
\hline
\end{tabular}

*Recalling Sentences sub-test

Ten TD controls (Mean age=17;9 years; range 14;6-21;9 years, 8 male) were recruited from central London via the Centre for DLDCN website or from newspaper and online community advertisements (such as gumtree.co.uk) and matched with the participants with SLI on Chronological Age (CA). The CA group were individually matched to the individual

\footnotetext{
${ }^{2}$ Ramus, Marshall, Rosen \& van der Lely (2013) tested 65 TD children aged between 5-12 years and reported mean z-scores of $0.06(S D=0.87)$ and $0.01(S D=0.77)$ for TAPS and VATT, respectively.
} 
with SLI to within 9 months of age) and they all had non-verbal IQ scores within the normal range $(\mathrm{RPM}:$ Mean=107.9, range=97-124).

Because of the late maturation of categorical perception in children (Hazan \& Barrett, 2000), ten additional Adult controls (Mean age $=27 ; 6$, range: 23;3-36;0 years, 3 male) were also recruited via adverts in online community websites to establish adult performance. None of the control participants taking part had any specific training in linguistics or speech sciences and none of them reported any history of speech and language therapy or familial history of language disorders. All participants (SLI, CA and Adults) were right-handed native speakers of British English and they all reported normal hearing. A one-way ANOVA for factor Age revealed that the three groups differed significantly $(F(2,29)=36.00, p<.001)$, and the post-hoc tests (Bonferroni corrected significance level, $\mathrm{p}=.017$ ) revealed that the participant ages in the two matched group (SLI and CA) were not significantly different $(\mathrm{p}=.521)$ whereas the Adult group differed significantly from both SLI and CA participants $(\mathrm{p}<.001)$

\section{Stimuli}

Two continua of seven synthetic CVC speech stimuli were created by using a Klatt type cascade-parallel formant synthesizer (HLsyn, Sensimetrics Inc., 1.0). The synthesis parameters closely resembled those previously used by Crowther \& Mann (1994) and Nittrouer (2004). (Note that the duration parameters were adjusted for British English based on earlier piloting of these tokens).

These CVC stimuli were identified as the English nonwords /bot/ and /bod/. This voiceless-voiced / $\mathrm{t}-\mathrm{d} /$ consonant contrast in English differs phonologically by one feature [voice] and it has only binary value, that is, it is either "on" or "off" ([+voice] or [-voice]). In 
English word-final stop consonants this phonological feature of [voice] is phonetically implemented in multiple acoustic cues, such as, in the duration of the preceding vocalic element and in the offset frequency of the first formant (F1). For example, in English, in the word-final voiced stop consonant [d] the preceding vowel is longer and the F1-offset frequency is lower than in the voiceless consonant [t] (Wolf, 1978).

These particular non-word stimuli were selected because i) the perception of the acoustic features contributing to the word-final stop consonant voicing has been previously studied with similar American English stimuli in TD children and adults (Crowther \& Mann, 1994; Nittrouer, 2004) and ii) instead of more commonly used word-initial contrasts (e.g., [ba]-[da]), these stimuli contain a perceptually less salient contrast where the formant transition is masked by the preceding vowel.

Two continua of these CVC words were synthesized to account for the English word final stop consonant voicing $([\mathrm{t}]-[\mathrm{d}])$. For all stimuli, the vocalic portions were preceded by $50 \mathrm{~ms}$ of silence (signalling the initial stop consonant $[\mathrm{b}]$ ) during which the amplitude of voicing (AV) parameter was interpolated from 40 to $60 \mathrm{~dB}$. The fundamental frequency $(\mathrm{F} 0)$ was set to increase from $100-130 \mathrm{~Hz}$ during the initial $50 \mathrm{~ms}$ after which it linearly decreased to $95 \mathrm{~Hz}$ to imitate a natural pitch contour in speech. In the voiceless token ([bot]) the F1 frequency was set to a constant value of $570 \mathrm{~Hz}$ throughout the word (F1-offset "high"). The voiced consonant [d] was created by lowering the offset frequency of $\mathrm{F} 1$ to $250 \mathrm{~Hz}$ during the final $50 \mathrm{~ms}$ (F1-offset "low"). In all stimuli, the F2 frequency was set to $1000 \mathrm{~Hz}$, rising to $1500 \mathrm{~Hz}$ during the final $50 \mathrm{~ms}$. The F3 frequency was kept at a constant value of $2650 \mathrm{~Hz}$. All higher formant frequencies (F4, F5, F6) were kept in the HLsyn default values (3500, $4500,4990 \mathrm{~Hz}$ respectively). Finally, a $15 \mathrm{~ms}$ linear onset and offset ramp was used to remove clicks and the amplitudes were normalized ( $\mathrm{rms}-10 \mathrm{~dB}$ of the maximum amplitude) by using CoolEdit96. All formant frequencies were checked using Praat (4.4.16; Boersma \& 
Weenink, 2006). For both [t] and [d], the vocalic duration changed from $100 \mathrm{~ms}$ to $220 \mathrm{~ms}$ in $20 \mathrm{~ms}$ steps (i.e., a total of seven steps; leading to total duration of the stimuli $150-280 \mathrm{~ms}$ ). Thus, each of the two continua ([t] and [d]: "high F1" and "low F1" respectively) contained seven different vocalic durations resulting in a total of 14 different stimuli.

\section{Procedure}

We adopted a traditional cue weighting paradigm where one acoustic parameter is fixed and the other parameter varies continuously (Nittrouer, Manning, \& Meyer, 1993; Nittrouer \& Studdert-Kennedy, 1987). The fixed property is the F1-offset (either "high F1" or "low F1", i.e., $570 \mathrm{~Hz}$ or $250 \mathrm{~Hz}$ ) and the continuously varying parameter is the duration of the vocalic portion (i.e., $100 \mathrm{~ms}$ to $220 \mathrm{~ms}$ in seven equal size steps).

The 14 synthetic stimuli used in the study were each played 10 times in a pseudo-random order in a two-alternative forced choice task (2-AFC). The stimuli were played on a laptop computer one sound at a time (SOA $1000 \mathrm{~ms}$; a total 140 stimuli) via headphones (Sennheiser) at a comfortable level (approximately $75 \mathrm{~dB}$ ). The participants were asked to identify the stimulus as the English non-word "bot" or "bod" by pressing a designated key (labelled with stickers) on the laptop keyboard. A short practice session of 15 category end-point stimuli, presented in a fixed order for all participants, preceded the experiment to establish that participants heard the stimuli as "bot" and "bod" and that they had understood the instructions. All participants achieved $80 \%$ or higher correct identification of these end-point stimuli in the practice session and were able to proceed to the actual experiment. The experimental task took approximately 15 minutes to complete.

All participants were tested individually in a quiet room at the Centre for

\section{DLDCN in UCL.}


The number of "bod" responses for each of the 14 stimuli was calculated. Then the sigmoid relationships were linearized by a probit transformation applied to the individual identification curves in each of the three groups (Cohen \& Cohen, 1983). In a phonetic categorisation task, category boundary is equivalent to a step in the continuum where the responses are expected to be at a chance level. This response inconsistency extends to tokens located near to the category boundary ('area of uncertainty') until preferential selection of one response category over the other is reached at the end of the stimulus continuum ('area of certainty'). A consistent response profile means that both areas of certainty $(20 \%$ and $80 \%)$ and uncertainty (35\% and 65\%) are located near the category boundary which means that participants reach certainty rapidly. The slope of the response function is equivalent to a change in probit units per change in the vocalic duration (ms) and it represents the degree of 'categoricalness' of the response.

In this study, three values that represent phonetic categorisation were derived from the identification data: i) location of the category boundary ( 0.5 probability level), ii) areas of certainty ( 0.2 and 0.8 probability levels $)$, and iii) areas of uncertainty ( 0.35 and 0.65 probability levels). All values were calculated by taking an average of the two synthetic continua (F1 high/low). In addition to these, two values that represent cue weighting were derived: i) weight assigned to the formant transition (F1-offset) and ii) weight assigned to the vocalic duration. To calculate the weight assigned to F1-offset, the separation between the two identification functions is calculated by subtracting the means of the category boundaries ( 0.5 probability level) of these two functions. The bigger the separation between the two boundary values, the greater is the weight assigned to F1-offset frequency. The mean slope of the two identification functions indicates how much weight is assigned to the vocalic duration. The steeper the slope, the greater is the weight assigned to vocalic duration. 
All analyses were conducted using SPSS (version 22.0, SPSS Inc., Chicago, IL). Main effects were considered significant for $\mathrm{p}<.05$. One-way ANOVAs with Group as a factor were performed separately for the category boundary value, measures of response consistency ('areas of certainty/uncertainty'), the mean of the slopes of the two identification functions ('weight assigned to vocalic duration'), and the boundary separation value between the two identification functions ('weight assigned to F1-offset'). All follow-up comparisons were corrected for multiple comparisons by using the Bonferroni method. Pearson correlations were performed to investigate the relationship between age, oral language skills, phonetic categorisation and cue weighting.

\section{Results}

The identification functions for the 30 participants and for each group are illustrated in Figure 1.

\section{Phonetic categorisation}

The values for the category boundary ( 0.50 probability level) and the response consistency (at $0.20,0.35,0.65,0.80$ probability levels) for the two identification functions 
CA

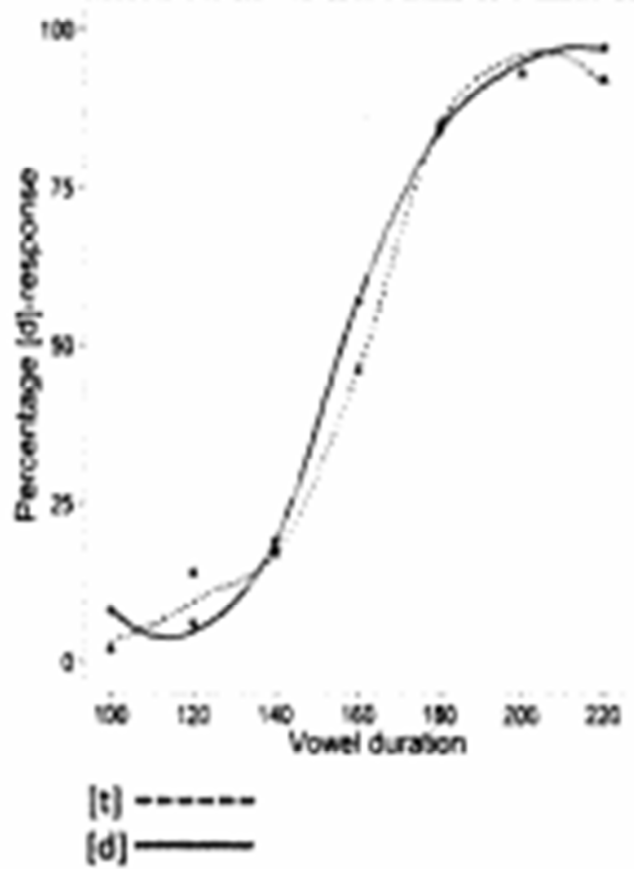

SU

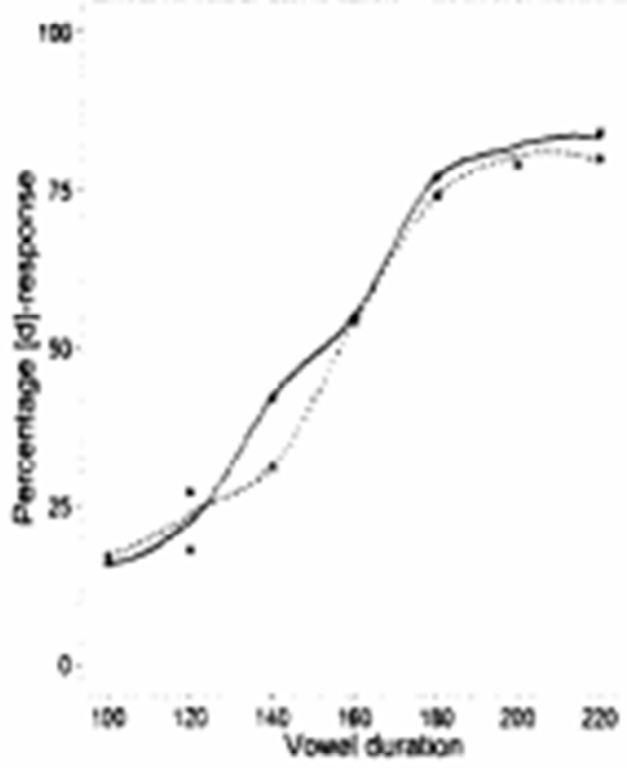

ADULTS

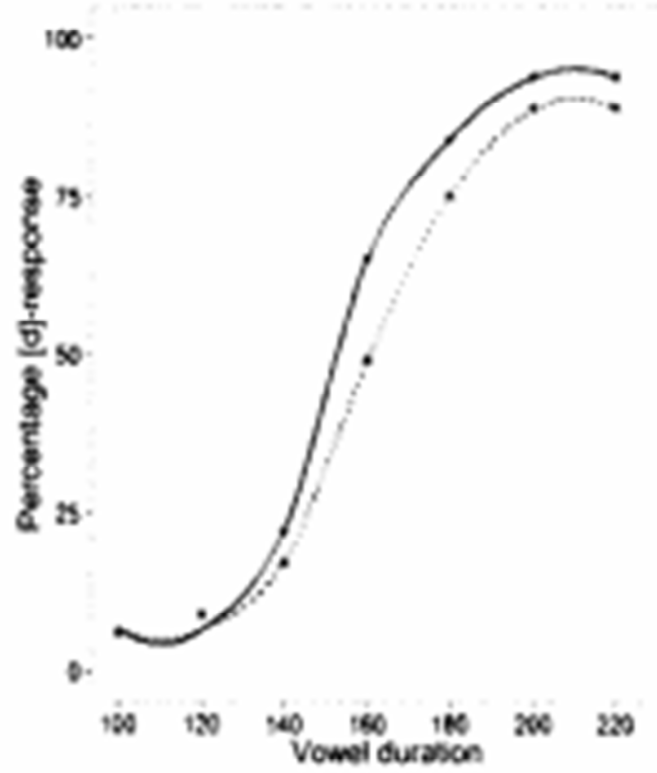

Figure 1. Identification functions (\% [d]-responses as a function of vocalic duration) for the two continua ([bot/d]) for the CA, SLI and nonmatched Adults. 
are presented in Table 2. A one-way ANOVA for the category boundary showed that the category boundaries did not differ between the groups $(F(2,27)=0.65, p=.531$; see Table 2 and Figure 1). All three groups placed the category boundary between $[\mathrm{t}]$ and $[\mathrm{d}]$ when the vocalic duration was at around $160 \mathrm{~ms}$ (i.e., at around step 4). However, a one-way ANOVA for the four response consistency measures showed that, when vocalic duration was long, adolescents with SLI reach certainty more slowly than both CA and Adult controls $(80 \%$ :

$\mathrm{F}(2,27)=6.73, \mathrm{p}=.004$; Post-hoc comparisons, $\mathrm{p}=.009$ and $\mathrm{p}=.002$ respectively; Bonferroni corrected significance level $\mathrm{p}=.017$ ). The two control groups did not differ significantly $(p=.693)$. When vocalic duration was short, the adolescents with SLI reached certainty marginally slower than the Adult controls $(20 \%$ : $F(2,27)=3.33, p=.051$; Post-hoc comparison: SLI versus Adults, $\mathrm{p}=.023$; all other comparisons $\mathrm{p}>.05$; Bonferroni corrected significance level $\mathrm{p}=.017$ ). For the group with SLI, vocalic duration of nearly $200 \mathrm{~ms}$ was required for $80 \%$ consistency in [d]-responses whereas both control groups preferred [d]-responses at and after $180 \mathrm{~ms}$ vowel duration. When the vowel was short, the control groups reached $80 \%$ consistency of [t]-responses (i.e., 20\% of [d]-responses) at approximately $130 \mathrm{~ms}$ whereas, for the group with SLI this level of consistency was reached only when the vocalic duration was less than $120 \mathrm{~ms}$. However, the performance at the two areas of uncertainty (35\% and $65 \%)$ did not differ between the three groups $(\mathrm{F}(2,27)=2.02, \mathrm{p}=.152$ and $(\mathrm{F}(2,27)=0.51, \mathrm{p}=.609$, respectively). This suggests that adolescents with SLI do not differ from CA and Adult controls in their response patterns at and around the category boundary $(35 \%, 50 \%$ and $65 \%)$ which all reflect areas of uncertainty. However, the group with SLI showed a preferential selection for one response category over the other category (at 20/80\% criterion levels) further away from the category boundary than both CA and Adult controls (at 80\%) and Adult controls (at 20\%). This indicates that the areas of uncertainty is bigger in the group with SLI than in the control groups. 
Table 2. Category boundaries for the two continua (high/low F1-offset), mean category boundary across the two continua (category boundary, $50 \%)$ and areas of certainty $(20 / 80 \%)$ and uncertainty $(35 / 65 \%)$ across the two continua.

\begin{tabular}{|l|l|l|l|l|l|l|l|}
\hline & $\begin{array}{l}\text { High } \\
\text { F1- } \\
\text { offset }\end{array}$ & $\begin{array}{l}\text { Low } \\
\text { F1- } \\
\text { offset }\end{array}$ & $\begin{array}{l}\text { Category } \\
\text { boundary } \\
(50 \%)\end{array}$ & $\begin{array}{l}\text { Area of } \\
\text { certainty } \\
(20 \%)\end{array}$ & $\begin{array}{l}\text { Area of } \\
\text { certainty } \\
(80 \%)\end{array}$ & $\begin{array}{l}\text { Area of } \\
\text { uncertainty } \\
(35 \%)\end{array}$ & $\begin{array}{l}\text { Area of } \\
\text { uncertainty } \\
(65 \%)\end{array}$ \\
\hline SLI & 3.58 & 3.88 & 3.73 & 1.71 & 5.76 & 2.81 & 4.63 \\
& $(1.35)$ & $(0.77)$ & $(0.99)$ & $(1.64)$ & $(0.66)$ & $(1.27)$ & $(0.79)$ \\
\hline CA & 4.06 & 3.76 & 3.91 & 2.69 & 5.11 & 3.36 & 4.50 \\
& $(0.40)$ & $(0.58)$ & $(0.27)$ & $(0.51)$ & $(0.33)$ & $(0.36)$ & $(0.25)$ \\
\hline Adults & 4.39 & 3.64 & 4.01 & 2.89 & 4.97 & 3.51 & 4.40 \\
& $(0.76)$ & $(0.51)$ & $(0.47)$ & $(0.78)$ & $(0.48)$ & $(0.52)$ & $(0.25)$ \\
\hline
\end{tabular}

Cue weighting

The steepness of the slope of the two identification functions, weight assigned to vocalic duration (mean of the slopes) and weight assigned to F1-offset frequency (boundary separation) are shown in Table 3.

Table 3. Cue weighting for the two synthetic continua: weight assigned to formant transition (boundary separation) and weight assigned to vocalic duration (slope mean). Standard deviation in brackets.

\begin{tabular}{|l|l|l|l|l|}
\hline GROUP & $\begin{array}{l}\text { High F1-offset } \\
\text { Slope }\end{array}$ & $\begin{array}{l}\text { Low F1-offset } \\
\text { Slope }\end{array}$ & $\begin{array}{l}\text { Weight assigned to } \\
\text { vocalic duration }\end{array}$ & $\begin{array}{l}\text { Weight assigned } \\
\text { to F1-offset }\end{array}$ \\
\hline SLI & $0.44(0.20)$ & $0.57(0.48)$ & $0.50(0.32)$ & $-0.30(0.97)$ \\
\hline AC & $0.71(0.28)$ & $0.75(0.30)$ & $0.73(0.25)$ & $0.30(0.84)$ \\
\hline Adults & $0.88(0.56)$ & $1.10(0.67)$ & $0.99(0.55)$ & $0.75(0.88)$ \\
\hline
\end{tabular}

A one-way ANOVA for the slope mean that reflects weight assigned to vocalic duration revealed a significant main effect of Group, $\mathrm{F}(2,27)=5.33, \mathrm{p}=.011$, and the post-hoc comparisons showed that the group with SLI had a significantly shallower slopes than Adults ( $p=.009$; Bonferroni corrected significance level $p<.017$; see table 3). However, the steepness of the mean of slopes of the two identification functions did not differ between the SLI and 
CA group nor did it differ between the CA and Adults (both comparisons, $p>.20$; see Table

3). The boundary separation measure reflects the weight assigned to the formant transition (F1-offset) did not differ significantly between the three groups, $F(2,27)=2.60, p=.093$.

These results indicate that adolescents with SLI assign less weight to vocalic duration than Adults. However, the group with SLI did not differ significantly from their CA matched controls nor do the CA controls differ from Adults. This pattern could indicate very subtle developmental delays in individuals with SLI in the use of vocalic length as a cue for word-final stop consonant voicing in English. Interestingly, our results show that adolescents with SLI do not differ from either control group on weight assigned to F1-offset frequency. This suggests that in this older group of individuals with SLI, processing rapid acoustic changes (such as formant transitions) is not impaired and that this cue is available to them, to the same degree as to both age-matched and adult controls, when making voicing judgements in word final consonants in English.

Because of the large age distribution in our SLI sample and the late maturation of categorical of perception, we ran four Pearson's correlations between chronological age, identification performance (two points of certainty) and weight assigned to vocalic duration (Bonferroni corrected significance level, $\mathrm{p}=.013$ ) separately for the three groups (Adults, $\mathrm{CA}$, $\mathrm{SLI})$. However, none of the comparisons reached significance ( $p>.2)$. In order to investigate the association between identification performance (two points of certainty), weight assigned to vocalic duration, and linguistic and nonlinguistic cognitive ability tapping vocabulary (BPVS-II, TWF-2), grammar (TROG-2, CELF-3, VATT, TAPS) and non-verbal IQ (RPM; see table 1), we ran 10 Pearson's correlations in the SLI group (Bonferroni corrected significance level, $p=.005)$. Again, none of the comparisons reached significance ( $>$ >.2). This suggests that the extended areas of uncertainty in phonetic categorisation tasks and less weight assigned to vocalic duration in cue weighting in the group with SLI compared to the 
control groups have little consequences on oral language skill. However, previous research has shown that children with SLI are heterogeneous with respect to their auditory and phonological abilities (Bishop \& Snowling, 2004; Rosen, Adlard \& van der Lely, 2009). Therefore, in addition to group differences, we were interested in investigating individual performance. We identified 'outliers' with abnormally low scores in our three tasks that showed significant group differences (areas of certainty at 0.2 and 0.8 level and weight assigned to vocalic duration) by using the procedure introduced by White et al. (2006). According to this procedure, the data is normalized using z-scores where the population mean $(\mu)$ and the standard deviation $(\sigma)$ are drawn from the matched CA control group. Those individuals scoring more than 1.65 standard deviations below ( 0.20 level and slope $)$ or above ( 0.80 level) the control mean are considered as impaired.
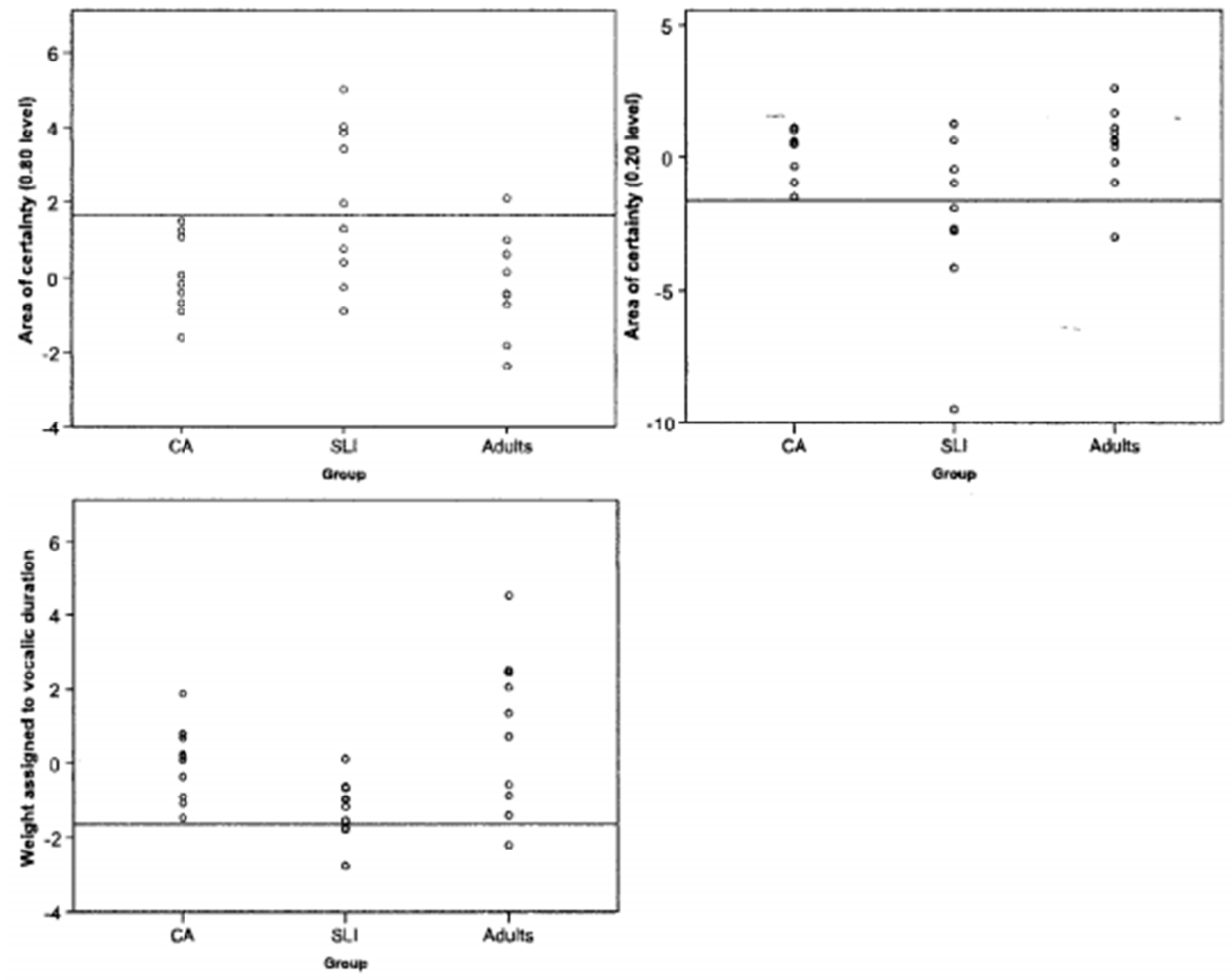

Figure 2. Individual z-scores for areas of certainty ( $80 \%$ and $20 \%$ identification) and weight assigned to vocalic duration (mean of the slopes of the two identification functions) for the CA, SLI and non-matched Adults. Solid line represents the chosen deviance threshold (1.65 $\mathrm{SD}$ above/below the CA mean). 
The analysis of the individual data indicates that, in the group with SLI, only two individuals (S03 and S09, see Appendix A) perform below the 1.65 cut-off for impaired performance in all three tasks. However, half of the individuals in the group with SLI (5/10 participants) reached both $20 \%$ and $80 \%$ certainty levels at a point that could be considered abnormally late in the continuum (see Figure 2) and 30\% of individuals with SLI were outside the 1.65 cut-off value in weight assigned to vocalic duration.

\section{Discussion}

In this study we investigated if adolescents and young adults with SLI weight the relevant acoustic cues for word-final stop consonant voicing in English to the same degree as their TD controls. Previous research has shown that children with SLI have difficulties in organising acoustic information to build phonetic categories in their L1 (Schwartz et al., 2013; Shafer et al., 2005; Sussman, 1993; 2001) and that they utilize some acoustic cues to a lesser degree than control children (Schwartz et al., 2013; Sussman, 1993). However, to our knowledge, no previous studies have directly contrasted two competing cues and investigated the relative weight assigned to each cue in individuals with SLI.

We employed a traditional categorical perception and cue weighting paradigm (Nittrouer et al., 1993) and manipulated two cues that are important for final stop consonant voicing in English: formant frequency transition and duration of the preceding vowel. We compared the performance of adolescents with SLI to their age-matched peers (CA group) and non-matched Adults. Based on previous findings, we predicted that, for phonetic categorisation measures, individuals with SLI would show greater variability in identification of unambiguous tokens compared with their age-matched controls (i.e. extended area of ambiguity) and shallower identification functions. For cue weighting, we predicted two 
possible outcomes: i) as individuals with SLI need more durational difference in vowels than TD controls to identify word final consonant voicing (Schwartz et al., 2013), we expected them to also assign less weight to this cue; ii) if individuals with SLI have a deficit in processing rapid auditory information (e.g. Tallal et al., 1993), we predicted that the formant frequency change cue would be less salient to them than the vocalic duration cue in this context.

As predicted, adolescents with SLI were less consistent in identifying the unambiguous tokens than both CA and non-matched Adult controls. Overall, adolescents with SLI needed more durational difference for the $80 \%$ accuracy level than the two control groups. In the ambiguous tokens the group with SLI did not differ from the controls. These finding replicate and extend the findings in younger children by Schwartz and colleagues (2013) and Sussman (1993) by showing that adolescents and young adults with SLI also show greater response variability and uncertainty for the good category representatives than TD individuals. For cue weighting, we found that adolescents and young adults with SLI assign less weight to vocalic duration as a cue for voicing than non-matched Adults (i.e. they had shallower identification slopes). This finding partially confirms our predictions. However, the fact that the group with SLI did not differ significantly from the CA group and that the CA group did not differ from the Adult group could potentially indicate a subtle developmental delay that could be better detected in younger individuals with SLI and by having a larger sample size. Lastly, we also showed that adolescents with SLI do not differ from TD controls on weight assigned to the formant transition cue. This finding suggests that the adolescents with SLI in the current sample do not have a deficit in processing acoustic cues that are brief in duration even when the cue is embedded in a less salient context (i.e. word final). Further evidence for this claim comes from the fact that significant differences between the group with SLI and the control groups were found for both short and long tokens (i.e. both ends of 
the continuum). Together these results suggest that adolescents and young adults with SLI have less accurate representations for good category members than the controls. In addition to this, they also weight vocalic duration as a cue for voicing less than Adult listeners, but they do not differ from the controls in weight assigned to the formant transition. However, Coady et al. (2005) have suggested that poor performance in a speech perception task in younger SLI children could be attributable to factors such as memory load, attention and failure to comply with the task instructions. This is also a valid point for the current study however, the fact that we found evidence for a speech perception deficit in an older group of individuals with SLI, who all found the task relatively easy, would support a genuine perceptual deficit in this group.

The analysis of the individual participants in the current study also supports previous findings in SLI by Rosen et al. (2009) that not all individuals with SLI show phonetic categorisation or cue weighting deficits. In those phonetic categorisation measures where we found significant group differences (i.e. unambiguous tokens) between SLI and controls, half of the individuals with SLI were associated with what counts as unimpaired scores, and in cue weighting, $70 \%$ of the group with SLI scored within the 1.65 SD cut-off criterion. This suggests that not all individuals with SLI necessarily have difficulties creating well defined phonetic categories and/or under-developed cue weighting strategies. Interestingly, our results showed that there is a substantial number of individuals with SLI who do show these deficits. In principle, this could mean that a sub-group of individuals with SLI have a deficit at the level of phonological representation and, that some of these individuals fail to correctly weight acoustic cues to phonological identity. However, as the participants in the current study were adolescents, there is an alternative explanation as the speech perception deficit may have been present during childhood in all of the individuals with SLI and resolved later in life for some of them. The finding in the current study of no 
direct relationship between phonetic categorisation, cue weighting and language skills supports the results found by Rosen and colleagues (2009). This could indicate that the presence of speech perception deficits, such as a phonetic categorisation deficit, has little impact on oral language skills and these might simply co-occur with the language deficits.

Overall, the findings of the current study provide further support for the notion that some individuals with SLI have less well defined phonological categories than their TD peers. As such, our results provide some support for previous findings by Nittrouer and colleagues (2011), Schwartz and colleagues (2013) and Sussman (1993). Moreover, contradictory findings in phonetic categorisation between previous results and the current study can also be attributed to differences in 'external' factors such as the age range of the participants, severity of language impairment, and task or stimulus design. For example, traditional categorical perception studies measure the change in perception as a function of acoustic change in a single cue (such as VOT). However, in cue weighting studies the effect of multiple cues for phonetic categorisation can be investigated. Adding cues increases the naturalness of the stimuli and is likely to have beneficial effects for phonetic labelling. Moreover, using natural speech tokens instead of synthetic stimuli is also likely to improve phonetic labelling.

We have shown that investigation of cue weighting can provide a valuable insight into the nature of phonetic categorisation skills in SLI and further research of cue weighting is warranted. Future research could focus on investigating age-effects (young versus older children with SLI) and contrasting multiple acoustic cues that are spectrally distinct and similar (Mayo \& Turk, 2005) using both synthetic and natural speech to help advance our understanding on speech perception deficits in SLI. 


\section{Acknowledgements}

The authors would like to thank the children and adults who took part in the study and members of the Centre for Developmental Language Disorders and Cognitive Neuroscience. We would also like to thank the two anonymous reviewers for their suggestions and comments.

Heather van der Lely died of cancer in February 2014, before this paper had been finalised for submission for publication. Heather van der Lely had a personal interest in the VATT and TAPS tests.

\section{Funding}

This research was supported by Wellcome Trust Grant (063713) awarded to Heather van der Lely and was carried out at the Centre for Developmental Language Disorders and Cognitive Neuroscience at UCL.

Declaration of interest: The authors report no conflicts of interest. The authors are responsible for the content and writing of the paper. 


\section{References}

Bishop, D.V.M. (1992). The Underlying Nature of Specific Language Impairment. Journal of Child Psychology and Psychiatry, 33, 3-66.

Bishop, D.V.M. (2003). The Test for Reception of Grammar, version 2 (TROG-2). Pearson Assessment, London.

Bishop, D.V.M., \& Snowling, M. (2004). Developmental dyslexia and specific language impairment: Same or different? Psychological Bulletin, 1, 858-886.

Boersma, P., \& Weenink, D. (2006). Praat: doing phonetics by computer (Version 4.4.16).

Coady, J.A., Kluender, K.R., \& Evans J.L. (2005). Categorical perception of speech by children with Specific Language Impairments. Journal of Speech, Language and Hearing Research, 48, 944-959.

Cohen, J., \& Cohen, P. (1983). Applied multiple regression/correlation analysis for the behavioral sciences. Hillsdale, N.J: L. Erlbaum Associates.

Crowther, C., \& Mann, V. (1994). Use of vocalic cues to consonant voicing and native language background: the influence of experimental design. Perception and Psychophysics, $55,513-525$.

Dorman, M.F., Studdert-Kennedy, M., \& Raphael, L.J. (1977). Stop- consonant recognition: Release bursts and formant transitions as functionally equivalent, contextdependent cues. Perception \& Psychophysics, 22, 109- 22.

Dunn, L.M., Dunn, L.M., Whetton, C., \& Burley, J. (1997). The British Picture Vocabulary Scales. 2nd edition. Windsor: NFER Nelson. 
German, D. (2000). Test of Word Finding-Second Edition. Austin, TX: Pro-Ed.

Gordon, P.C., Eberhardt, J.L., \& Rueckl, J.G. (1993). Attentional Modulation of the Phonetic Significance of Acoustic Cues. Cognitive Psychology, 25, 1-42.

Hazan, V., \& Barrett, S. (2000). The development of phonemic categorization in children aged 6-12. Journal of Phonetics, 28, 377-396.

Holt, L.L., \& Lotto, A.J. (2006). Cue weighting in auditory categorization: Implications for first and second language acquisition. Journal of the Acoustical Society of America, 119, 3059-3071.

Jusczyk, P.W., \& Luce, P.A. (1994). Infants' Sensitivity to Phonotactic Patterns in the Native Language Journal of Memory and Language, 33, 630-645.

Krause, S.E. (1982). Vowel duration as a perceptual cue to postvocalic consonant voicing in young children and adults. Journal of the Acoustical Society of America, 71, 990995.

Kuhl, P.K., Williams, K.A., Lacerda, F., Stevens, K.N., \& Lindblom, B. (1992). Language experience alters phonetic perception in infants by 6 months of age. Science, 255, 606-608.

Leonard, L. (2014). Children with specific language impairment, $2^{\text {nd }}$ Edition. Cambridge, Mass.: MIT Press.

Liberman, A. M., Harris, K. S., Hoffman, H. S., \& Griffith, B. C. (1957). "The discrimination of speech sounds within and across phoneme boundaries". Journal of Experimental Psychology, 54, 358-368. 
Mayo, C., \& Turk, A. (2004). Adult-child differences in acoustic cue weighting are influenced by segmental context: children are not always perceptually biased toward transitions. The Journal of the Acoustical Society of America, 115, 3184- 3194.

Mayo, C., \& Turk, A. (2005). The influence of spectral distinctiveness on acoustic cue weighting in children's and adults' speech perception. The Journal of the Acoustical Society of America, 118, 1730-1741.

Morrongiello, B.A., Robson, R.C., Best, C.T., \& Clifton, R.K. (1984). Trading relations in the perception of speech by five-year-old children. Journal of Experimental Child Psychology, 37, 213-250.

Nittrouer, S. (1992). Age-related differences in perceptual effects of formant transitions within syllables and across syllable boundaries. Journal of Phonetics, 20, 351-382.

Nittrouer, S. (2004). The role of temporal and dynamic signal components in the perception of syllable-final stop voicing in children and adults. Journal of the Acoustical Society of America, 115, 1777-1790.

Nittrouer, S. (2005). Age-related differences in weighting and masking of two cues to word-final stop voicing in noise. The Journal of the Acoustical Society of America, 118, 10721088.

Nittrouer, S, Crowther, C., \& Miller, M. (1998). The relative weighting of acoustic properties in the perception of (s)+stop clusters by children and adults. Perception and Psychophysics, 60, 51-64.

Nittrouer, S., Manning, C., \& Meyer, G. (1993). The perceptual weighting of acoustic cues changes with linguistic experience. The Journal of the Acoustical Society of America, 94, S1865. 
Nittrouer, S., \& Miller, M. (1997). Predicting developmental shifts in perceptual weighting schemes. The Journal of the Acoustical Society of America, 101, 2253-2266.

Nittrouer, S., Miller, M., Crowther, C., \& Manhart, M. (2000). The effect of segmental order on fricative labeling by children and adults. Perception and Psychophysics, 62, 266284.

Nittrouer, S., Shune, S., \& Lowenstein, J. H. (2011). What is the deficit in phonological processing deficits: Auditory sensitivity, masking, or category formation? Journal of Experimental Child Psychology, 108, 762-785.

Nittrouer, S., \& Studdert-Kennedy, M. (1987). The role of coarticulatory effects in the perception of fricatives by children and adults. Journal of Speech and Hearing Research, 30, 319-329.

Ohde, R.N., Haley, K.L., Vorperian, H.K., \& McMahon, C.W. (1995). A developmental study of the perception of onset spectra for stop consonants in different vowel environments. The Journal of the Acoustical Society of America, 97, 3800-3812.

Ramus, F., Marshall, C.R., Rosen, S., \& van der Lely, H.K.J. (2013). Phonological deficits in specific language impairment and developmental dyslexia: towards a multidimensional model. Brain, 136, 630-645.

Raven, J., Raven, J. C., \& Court, J. H. (1998). Raven's progressive matrices. Oxford: Oxford Psychologists Press.

Rosen, S., Adlard, A., \& van der Lely, H.K.J. (2009). Backward and Simultaneous Masking in Children with Grammatical Specific Language Impairment: No Simple Link Between Auditory and Language Abilities. Journal of Speech Language and Hearing Research, 52, 396-411. 
Semel, E.M., Wiig, E.H., \& Secord, W. (1995). Clinical Evaluation of Language Fundamentals R-3. San Antonio, TX: The Psychological Corporation.

Shafer, V.L., Morr, M.L., Datta, H., Kurtzberg, D., \& Schwartz, RG. (2005). Neurophysiological Indexes of speech processing deficits in children with Specific Language Impairment. Journal of Cognitive Neuroscience, 17, 1168- 1180.

Sussman, J.E. (2001). Vowel perception by adults and children with normal language and specific language impairment: Based on steady states or transitions? The Journal of the Acoustical Society of America, 109, 1173-1180.

Sussman, J.E. (1993). Perception of formant transition cues to place of articulation in children with language impairments. Journal of Speech and Hearing Research, 36, 12861299.

Schwartz, R.G., Scheffler, F., \& Lopez, K. (2013). Speech perception and lexical effects in specific language impairment. Clinical Linguistics and Phonetics, 27, 339-354.

Tallal, P. (1976). Rapid auditory processing in normal and disordered language development. Journal of Speech and Hearing Research, 19, 561-571.

Tallal, P., Miller, S., \& Fitch, R. (1993). Neurobiological basis of speech: a case for the preeminence of temporal processing. Annals of New York Academy of Sciences, 682, 2747.

Tallal, P., \& Piercy, M. (1975). Developmental aphasia: The perception of brief vowels and extended stop consonants. Neuropsychologia, 13, 69-74. 
Tallal, P., \& Stark, R. (1981). Speech acoustic cue discrimination abilities of normally developing and language impaired children. The Journal of the Acoustical Society of America, $69,568-574$.

van der Lely, H.K.J. (2000). Verb Agreement and Tense Test (VATT). Available from www.dlden.com.

van der Lely, H. K. J. (1996a) The Test of Active and Passive Sentences (TAPS). Available from www.dlden.com.

van der Lely, H.K.J. (1996b). Specifically language impaired and normally developing children: Verbal passive vs. adjectival passive sentence interpretation. Lingua, 98, 243-272.

van der Lely, H.K.J., Rosen, S., \& McClelland, A. (1998). Evidence for a Grammar specific deficit in children. Current Biology, 8, 1253-1258.

Werker, J., \& Tees, R.C. (1984). Cross-language speech perception: evidence for perceptual reorganization during the first year of life. Infant behaviour and development, 7 , 49-63.

White, S., Milne, E., Rosen, S., Hansen, P., Swettenham, J., Frith, U., \& Ramus, F. (2006). The role of sensorimotor impairments in dyslexia: A multiple case study of dyslexic children. Developmental Science, 9, 237-255.

Wolf, C.G. (1978). Voicing cues in English final stops. Journal of Phonetics, 6, 299309. 


\section{Appendix.}

Details of SLI participants scores on standardised (in z-scores) and non-standardised (in \%correct) at the time of selection.

\begin{tabular}{|c|c|c|c|c|c|c|c|}
\hline Participant & RPM & TROG-2 $^{\mathrm{a}}$ & BPVS-II $^{\mathrm{b}}$ & CELF-3 $^{\mathrm{c}}$ & TWF-2 $^{d}$ & VATT & TAPS $^{\mathrm{e}}$ \\
\hline S01 & -0.73 & -0.33 & -1.53 & -2.00 & -1.40 & 80 & 69 \\
\hline S02 & -0.80 & -2.20 & -2.07 & -2.33 & -2.27 & 5 & 48 \\
\hline S03 & -0.33 & -0.20 & -2.33 & -2.00 & -1.80 & 30 & 46 \\
\hline S04 & -0.27 & -0.33 & -1.27 & N/A & -1.40 & 73 & 65 \\
\hline S05 & 0.20 & -1.93 & -2.73 & -2.33 & N/A & 38 & 67 \\
\hline S06 & 0.07 & -1.93 & -2.00 & -2.33 & -1.87 & 65 & 40 \\
\hline S07 & -0.90 & -2.07 & -2.33 & -2.33 & -2.60 & 3 & 46 \\
\hline S08 & -0.27 & -0.80 & -0.67 & -2.33 & -1.33 & N/A & 58 \\
\hline S09 & 0.27 & -0.80 & -1.87 & -2.33 & -1.13 & 75 & 52 \\
\hline S10 & 0.40 & -1.23 & -1.87 & -2.00 & -1.40 & 35 & 73 \\
\hline Mean & -0.24 & -1.18 & -1.87 & -2.22 & -1.69 & 44.89 & 56.40 \\
\hline$S D$ & 0.47 & 0.79 & 0.59 & 0.17 & 0.49 & 29.68 & 11.54 \\
\hline
\end{tabular}

Z-scores of -1.5 (or lower) and an accuracy of $80 \%$ (or lower) are highlighted in grey.

Notes:

${ }^{a}$ TROG-2 is standardised on children up to the age of 16.0, for children older than 16.0 their raw scores were converted to standard scores at the ceiling level for a 16 year old.

${ }^{\mathrm{b}}$ BPVS-II is standardised on children up the age of $15 ; 8$, for children older than $15 ; 8$ their raw scores were converted to standard scores at the ceiling level for a 15;8 year old.

${ }^{\mathrm{c}}$ CELF-3 Recalling sentences subtest is standardised on children up to the age of 21.0, for participants older than 21.0 their raw scores were converted to standard scores at the ceiling level for a 21 year old.

${ }^{3}$ TWF-2 is standardised on children up the age of $12 ; 11$, for children older than $12 ; 11$ their raw scores were converted to standard scores at the ceiling level for a 12;11 year old.

${ }^{4}$ TAPS - reports correct scores for Full Active, Full Passive and Short Passive Sentences. 